\author{
Moosa DARIJANI, PhD Candidate \\ E-mail: Moosa.darijani@yahoo.com \\ Department of Industrial Management \\ Faculty of Management and Accounting \\ Allameh Tabataba'i University, Tehran, Iran \\ Professor Maghsoud AMIRI, PhD (Corresponding author) \\ E-mail: mg_amiri@yahoo.com \\ Department of Industrial Management \\ Faculty of Management and Accounting \\ Allameh Tabataba'i University, Tehran, Iran \\ Associate Professor Mohammad Taghi TAGHAVIFARD, PhD \\ E-mail: dr.taghavifard@gmail.com \\ Department of Industrial Management \\ Faculty of Management and Accounting \\ Allameh Tabataba'i University, Tehran, Iran \\ Associate Professor Payam HANAFIZADEH, PhD \\ E-mail: hanafizadeh@gmail.com \\ Department of Industrial Management \\ Faculty of Management and Accounting \\ Allameh Tabataba'i University, Tehran, Iran
}

\title{
EFFICIENCY ASSESSMENT USING COMMON-WEIGHT FDEA UNDER A MULTI-OBJECTIVE APPROACH AS WELL AS POSSIBILITY AND NECESSITY THEORY
}

Abstract. In the present study, we introduce a novel method for evaluating Decision-Making Units (DMUs) in Fuzzy Data Envelopment Analysis (FDEA). Initially, the problem was modeled using common weights in Data Envelopment Analysis (DEA). Then, the model was developed using the possibility and necessity theory to obtain common weights for each input and output of the DMUs. Next, the performance of DMUs was evaluated. Since the results were fuzzy numbers, first, we adopted a ranking method to convert them into crisp numbers and then, compared them with each other. In the next step, ranking of the DMUs was carried out. Finally, a practical example was solved via the developed method.

Keywords: Fuzzy Data Envelopment Analysis, Common Weights, Possibility Theory, Necessity Theory, Fuzzy Number Ranking.

JEL Classification : C02- C52- C61- O14- G11

DOI: $10.24818 / 18423264 / 55.3 .21 .08$ 
Moosa Darijani, Maghsoud Amiri, Mohammad Taghi Taghavifard, Payam Hanafizadeh

\section{Introduction}

Charnes, Cooper, and Rhodes developed Data Envelopment Analysis (DEA) and their model was acronymed CCR (Charnes, A et al, 1978). DEA provides a procedure for evaluating the relative efficiency of Decision-Making Units (DMUs) in complexes such as universities, manufactories, and stores. Although DEA distinguishes efficient DMUs from inefficient ones, this method can provide ranking only for the latter, not the efficient DMUs (Jahanshahloo et al, 2010). Since in the CCR model, different weights are used for the input and output of various DMUs, real-time performance evaluation requires a general view of the relative importance of input and output ( $\mathrm{Li}$ et al, 2007). However, some problems may arise when using a classic DEA for ranking. First of all, the scores obtained by the DEA do not sort the DMUs completely and some of the categorized DMUs are usually inefficient. This weakness is obvious in DEA application, especially when the total number of input and output is larger than the number of DMUs. To tackle this shortcoming, different approaches have been proposed (Hosseinzadeh et al, 2013). In addition, the use of different weights in the evaluation of a DMU is undesirable since it prevents the flexibility of comparison between DMUs based on common weights (Kao and Hung, 2005). Of note, although some DMUs may have better performance than those with efficiency scores equal to one, they are classified as inefficient when they take inappropriate weights due to the maximum performance of other DMUs (Dyson and Thanassoulis, 1988). Accordingly, some researchers do not find calculating different weights for the same criterion in a set of similar DMUs logical, hence their search for ways to calculate common weights of input and output parameters of the model. In this regard, Roll et al. introduced the topic of common weights for the first time (Roll et al,1991).

Although the DEA is a proper tool to calculate efficiency of DMUs, it is still faced with some considerable constraints, one of the most important ones being its sensitivity to data as it focuses on the boundary or interval. As a result, errors in the measurement of data cause major problems and successful application of the DEA method depends on finding the exact input and output values. This is while, in some cases, due to complexity of the input and output, it is difficult to obtain precise, unambiguous data. Furthermore, the available data used in performance analysis are often qualitative and linguistic, e.g., "old equipment" and "good" service (Lertworasirikul et al, 2003).

In recent years, different approaches to the quantification of ambiguous and inaccurate data have been presented. Fuzzy sets theory is an instance of methods recommended for use in DEA models Zimmermann (1996). Generally, Fuzzy Data Envelopment Analysis (FDEA) models are more accurate than conventional methods in dealing with real-world problems. Fuzzy sets theory uses linguistic data in DEA models. On the other hand, FDEA models are supposed to 
Efficiency Assessment Using Common-weight FDEA under a Multi-objective Approach as well as Possibility and Necessity Theory

use fuzzy Linear Programming (LP) models. There are different fuzzy ranking methods in fuzzy LP models that may generate various results. Therefore, ranking fuzzy sets requires using a proper method. Many researchers have addressed fuzzy sets problems in their studies Zimmermann (1996). A study by Emrouznejad et al. reviewed the FDEA methods published in the last two decades and classified them into six primary categories: tolerance method, basis $\alpha$-level method, fuzzy ranking method, possibility method, fuzzy mathematical method, and the fuzzy sets random / type-2 method. Also, it presented a secondary ranking for a pioneering group of articles which did not fall into the first six classifications. In most studies applying the above-mentioned approaches, fuzzy models have been transformed into classical LP or interval models (Emrouznejad et al, 2014). One among such methods is the possibility theory, which is a mathematical theory for ambiguous numbers. It is well known as an appropriate and integrated theory for analyzing uncertainties in a decision-making environment (Dubois and Prade, 2001). When applying the possibility theory, the model should be transformed into LP (Dubois and Prade, 1988), Liu (1999).

In this paper, a novel multi-objective fuzzy DEA efficiency evaluation approach is presented. In the developed model, common weights for each input and output of the DMUs for their evaluation can be calculated using the possibility and necessity theory. The efficiency calculated for each DMU is a fuzzy number. Therefore, a fuzzy numbers ranking method is used to convert the fuzzy numbers into crisp ones and then, they are compared with each other.

In the present paper, a brief discussion of the DEA, common-weight DEAMCDM method, fuzzy DEA, possibility and necessity theory, and triangular fuzzy numbers ranking approach is given in Section 2. Section 3 is devoted to the integration of the possibility and necessity theory into the common-weight DEAMCDM. A practical example is solved to show applicability of the proposed model. The paper is summarized and concluded and some directions for future research are provided in Section 4.

\section{Methodology:}

Possibility and necessity theory integrated into DEA and common-weight DEA-MCDM

\subsection{Preliminaries}

\subsubsection{Data Envelopment Analysis Method}

CCR model is commonly used in the studies on DEA. Suppose that there are $n$ DMUs with equal numbers of input and output. If each DMU has $r$ input and s output, then $x_{i j}$ and $y_{i k}$ indicate the value of the $\mathrm{j}$-th input and the value of the kth output for the $\mathrm{i}$-th DMU, respectively; $\mathrm{i}=1 . \cdots . \mathrm{n}, j=1 . \cdots . r, k=1 . \cdots . s$. 
Moosa Darijani, Maghsoud Amiri, Mohammad Taghi Taghavifard, Payam Hanafizadeh

Table 1. Defining data

\begin{tabular}{|c|c|c|c|c|c|c|}
\hline DMUs & Inputs & & & Outp & & \\
\hline 1 & $x_{11}$ & $\ldots$ & $x_{1 r}$ & $y_{11}$ & $\cdots$ & $y_{1 s}$ \\
\hline 2 & $x_{21}$ & $\cdots$ & $x_{2 r}$ & $y_{21}$ & $\cdots$ & $y_{1 s}$ \\
\hline$\vdots$ & $\vdots$ & $\cdots$ & $\vdots$ & $\vdots$ & $\cdots$ & $\vdots$ \\
\hline $\mathrm{n}$ & $x_{n 1}$ & $\cdots$ & $x_{n r}$ & $y_{n 1}$ & $\begin{array}{c}\cdots \\
y_{i}^{t}\end{array}$ & $y_{n s}$ \\
\hline
\end{tabular}

Similarly, $x_{i}$ and $y_{i}$ are r-dimensional input and s-dimensional output vectors of unit $i$, respectively, as shown in Table (1). Also, $v^{q}$ and $u^{q}$ are output weight and input weight vectors of a specifiedDMU $\mathrm{U}_{q}$, respectively, and $q \in$ $\{1 \ldots . . n\}$. To evaluate the performance of each DMU, the CCR model can be used as follows (Carrillo et al, 2016), (Charnes et al, 1978):

$\max E_{q}=\frac{y_{q}^{t} v^{q}}{x_{q}^{t} u^{q}}$

s.t

$\frac{y_{i}^{t} v^{q}}{x_{i}^{t} u^{q}} \leq 0 \quad$ for all $i=1$....n

$u^{q} \geq \varepsilon_{r} \cdot v^{q} \geq \varepsilon_{s}$

Where $\varepsilon_{r}$ and $\varepsilon_{s}$ are vectors of small positive numbers that keep weights away from zero. The above model is a nonlinear programming model that can be converted into an LP model as follows:

$\max y_{q}^{t} v^{q}$

s.t

$x_{q}^{t} u^{q}=1$

$y_{i}^{t} v^{q}-x_{i}^{t} u^{q} \leq 0$ for all $i=1 \ldots . . n$

$u^{q} \geq \varepsilon_{r} \cdot v^{q} \geq \varepsilon_{s}$

The above model has to be solved $\mathrm{n}$ times to obtain efficiency for each DMU among n DMUs.

\subsubsection{Fuzzy Data Envelopment Analysis (FDEA) Method}

Fuzzy sets theory is an appropriate approach to the quantification of ambiguous and inaccurate input and output data in the DEA model. Suppose that there are $\mathrm{n}$ DMUs with the numbers of their input and output being equal, where each DMU has $\mathrm{r}$ input and s output. Input and output data are fuzzy numbers and $\tilde{x}_{i}^{t}$ and $\tilde{y}_{i}^{t}$ indicate their measures, respectively. $v^{q}$ and $u^{q}$ are also vectors of output weights and input weights for a specified $\mathrm{DMU}_{q}$ and $q \in\{1 \ldots . n\}$. Therefore, to evaluate the performance of each DMU, the fuzzy coefficient CCR (FCCR) can be defined as follows:

(FCCR) $\max \tilde{y}_{q}^{t} v^{q}$ 
Efficiency Assessment Using Common-weight FDEA under a Multi-objective Approach as well as Possibility and Necessity Theory

$\tilde{x}_{q}^{t} u^{q}=1$

$\tilde{y}_{i}^{t} v^{q}-\tilde{x}_{i}^{t} u^{q} \leq 0$ for all $i=1 \ldots . n$

$u^{q} \geq \varepsilon_{r} \cdot v^{q} \geq \varepsilon_{s}$

$\varepsilon_{r}$ and $\varepsilon_{s}$ are introduced to Equation (1). Again, the above model has to be solved $\mathrm{n}$ times to obtain efficiency for each DMU among $\mathrm{n}$ DMUs.

\subsubsection{DEA-MCDM Common Weight Method}

Common-weight DEA-MCDM approach was introduced by (Carrillo et al, 2016). In this approach, the non-negative weight vectors $u \in I R^{r}$ and $v \in I R^{S}$ are considered. $x_{i}^{t} u$ and $y_{i}^{t} v$ show the corresponding sums of input and output values for the $i$-th DMU. The maximum efficiency ratio of $y_{i}^{t} v / x_{i}^{t} u$ can be obtained by minimizing $x_{i}^{t} u$ and simultaneously maximizing $y_{i}^{t} v$. Note that for input and output weights of each DMU, there exists a pair $\left(x_{i}^{t} u . y_{i}^{t} v\right)$ associated with the sums of input and output spaces as shown in Figure (1) (Carrillo et al, 2016). In a Compromise Programming (CP) paradigm, each DMU is presented by a point near the ideal with the sums of weighted input and weighted output (left upper corner).
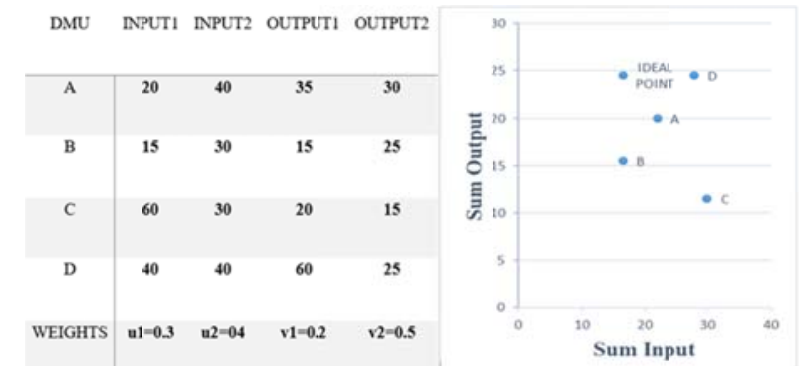

Figure 1. Each DMU on the sum of weighted input/output space

In this case, different sets of weights create various schemes with different options collected in a desirable position. The aim is finding common weights to put each $n$ DMUs in the closest possible position to an ideal point with the minimum sum of weighted input and the maximum sum of weighted output through a CP approach. For each special (u.v)-dependent bi-dimensional space symptom, a distance to the ideal point can be considered for the bi-objective problem. To model the above assumptions, the ideal points of the sum input and sum output are formulated as follows:

$\left(I_{x}(u) \cdot I_{y}(v)\right)=\left(\min x_{i}^{t} u . \max y_{i}^{t} v\right) i=1 \ldots . n$

Also, the distance between points $\left(I_{x}(u) . I_{y}(v)\right) \epsilon I R^{2}$ corresponding to the ideal point and the $\mathrm{i}$-th DMU is considered in lp-norm as follows:

$D_{i}(u . v)=\left(\left(x_{i}^{t} u-I_{x}(u)\right)^{p}+\left(I_{y}(v)-y_{i}^{t} v\right)^{p}\right)^{1 / p}$ 
Moosa Darijani, Maghsoud Amiri, Mohammad Taghi Taghavifard, Payam Hanafizadeh

This method searches for common weights of $u$ and $v$ that locate each $n$ mapped DMU in the closest possible position to the ideal points in the sum input and sum output, simultaneously. Note that it does not give the best searched DMU, but achieves the best common weights that put all the $\mathrm{n}$ DMUs in the best positions. Hence, a multi-objective problem is selected as follows (Carrillo et al, 2016):

vmin $\left(D_{1}(u . v) \ldots . D_{n}(u . v)\right)$

s.t

$y_{i}^{t} v-x_{i}^{t} u \leq 0 \quad$ for all $i=1 . \ldots . n$

$u \geq \varepsilon_{r} \cdot v \geq \varepsilon_{s}$

Among various available approaches to multi-objective problems, the weighting procedure is used to minimize a weighted sum of the problem objectives. Thus, the weights of $\mathrm{n}$ objectives are equal since the importance of all DMUs is considered the same. Accordingly, the following model is solved:

$\min \sum_{i=1}^{n} D_{i}(u . v)$

$y_{i}^{t} v-x_{i}^{t} u \leq 0$ for all $i=1 . \ldots . n$

$u \geq \varepsilon_{r} \cdot v \geq \varepsilon_{s}$

It is clear that the above model requires a nonlinear solution approach as the functions are minimized and maximized to calculate $D_{i}$. Thus, a proper modification to the model makes the use of linear optimization approaches easier for $\mathrm{p}=1$.

$D_{i}(u . v)=\left(x_{i}^{t} u-I_{x}(u)\right)+\left(I_{y}(v)-y_{i}^{t} v\right)$

It is obvious that Equation (5) is convertible to the following model.

$\min \sum_{i=1}^{n}\left(x_{i}^{t} u-m\right)+\left(M-y_{i}^{t} v\right)$

$m-x_{i}^{t} u \leq 0$ for all $i=1$.....n

$M-y_{i}^{t} v \geq 0 \quad$ for all $i=1 . \ldots . n$

$y_{i}^{t} v-x_{i}^{t} u \leq 0$ for all $i=1 . \ldots . n$

$u \geq \varepsilon_{r} \cdot v \geq \varepsilon_{s}$

M. $m \geq 0$

$\varepsilon_{r}$ and $\varepsilon_{s}$ are introduced to Equation (1) (Carrillo et al, 2016).

In general, calculation of efficiency is done in two steps as follows:

Initially, Model (6) is solved to obtain the weights of the input (minimization) and output (maximization) criteria $u^{*} . v^{*}$

The efficiency of each unit can be calculated as follows:

$E_{q}=\frac{y_{q}^{t} v^{*}}{x_{q}^{t} u^{*}}$

\subsubsection{Integrated Possibility Theory and DEA Model}

The possibility approach was proposed to compute an FCCR model by Lertworasirikul et al. (2003). The FCCR model is converted to a CCR model by the possibility concepts as fuzzy coefficients. FCCR can be formulated as the following model, called the possibility CCR (PCCR) model: 
Efficiency Assessment Using Common-weight FDEA under a Multi-objective Approach as well as Possibility and Necessity Theory

\begin{tabular}{l}
\hline$(P C C R) \quad \max \quad \bar{f} \cdot \bar{f}$ \\
$\pi\left(\tilde{y}_{q}^{t} v^{q} \geq \bar{f}\right) \geq \beta(\mathrm{a})$ \\
$\pi\left(\left(\tilde{x}_{q}^{t} u^{q}=1\right) \geq \alpha_{\circ}(b)\right.$ \\
$\pi\left(\tilde{y}_{i}^{t} v^{q}-\tilde{x}_{i}^{t} u^{q} \leq 0\right) \geq \alpha \quad$ for all $i=1 . \ldots . n(c)$ \\
$u^{q} \geq \varepsilon_{r} \cdot v^{q} \geq \varepsilon_{s}$
\end{tabular}

In the above model, $\beta$ and $\alpha_{0} \in[0.1]$ are assumed as the acceptable values for the constraints (a) and (b), respectively. Furthermore, $\alpha=\left[\alpha_{\circ} \ldots \ldots . \alpha_{n}\right]^{T} \in$ $[0 ; 1]^{n}$ is assumed a column of the allowable values for the possibility constraint (c).

This means that the optimal $\bar{f}$ is calculated with the maximum value so that the level of the constraints (a), (b), and (c) reaches at least the values of . $\alpha$ 。 and $\alpha$. Also, the value of $\tilde{y}_{q}^{t} v^{q}$ is calculated greater than or equal to $\bar{f}$ with the possibility level $\beta$ such that it does not exceed the specified limit for the constraints (Lertworasirikul et al, 2003). At the possibility level $\alpha$, efficient DMU and inefficient DMU are defined as follows:

Definition: A DMU is efficient at the possibility level $\alpha$ if and only if the value of $\bar{f}$ is greater than or equal to one; otherwise, the DMU is inefficient at the possibility level $\alpha$.

The following lemma is used to solve the above model;

Suppose that $\tilde{r}_{1} \cdot \tilde{r}_{2} \cdot \tilde{r}_{3} \ldots \ldots . \tilde{r}_{n}$ are fuzzy variables defined as fuzzy sets with convex and normal membership functions $(\cdot)_{\alpha_{i}}^{L}$ and $(\cdot)_{\alpha_{i}}^{U}$ being the lower and upper bounds for the $\alpha$-level fuzzy variables $\tilde{r}_{i}, \mathrm{i}=1 \ldots . \mathrm{n}$. (Figure 1 ) at the possible levels $\alpha_{2} . \alpha_{1}$ and $\alpha_{3} 0 \leq \alpha_{1} \cdot \alpha_{2} . \alpha_{3} \leq 1$, respectively.

$\pi\left(\tilde{r}_{1}+\cdots+\tilde{r}_{n} \leq b\right) \geq \alpha \quad$ if and only if

$\left(\tilde{r}_{1}\right)_{\alpha_{1}}^{L}+\cdots+\left(\tilde{r}_{n}\right)_{\alpha_{1}}^{L} \leq b$

$\pi\left(\tilde{r}_{1}+\cdots+\tilde{r}_{n} \geq b\right) \geq \alpha \quad$ if and only if

$\left(\tilde{r}_{1}\right)_{\alpha_{1}}^{U}+\cdots+\left(\tilde{r}_{n}\right)_{\alpha_{1}}^{U} \geq b$

If input and output numbers in the PCCR model are convex and normal, according to Equation (9), the PCCR model is written as follows:

$$
\begin{aligned}
& (P C C R) \quad \max \quad \bar{f} \\
& \left(\tilde{y}_{q}^{t} v^{q}\right)_{\beta}^{R} \geq \bar{f} \\
& \left(\tilde{x}_{q}^{t} u^{q}\right)_{\alpha \circ}^{R} \geq 1 \\
& \left(\tilde{x}_{q}^{t} u^{q}\right)_{\alpha_{\circ}}^{L} \leq 1 \\
& \left(\tilde{y}_{i}^{t} v^{q}-\tilde{x}_{i}^{t} u^{q}\right)_{\alpha}^{L} \leq 0 \quad \text { for all } i=1 . \ldots . n \\
& u^{q} \geq \varepsilon_{r} \cdot v^{q} \geq \varepsilon_{s}
\end{aligned}
$$


Moosa Darijani, Maghsoud Amiri, Mohammad Taghi Taghavifard, Payam Hanafizadeh

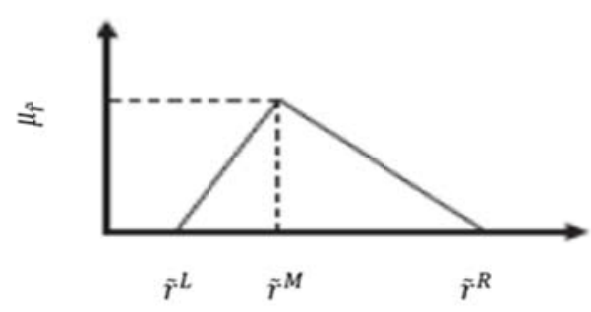

Figure 2. membership function of a triangular fuzzy number

Using Lemma (1) introduced by Liu (1999) for triangular fuzzy numbers $\tilde{r}_{i}=\left(\left(\tilde{r}_{i}\right)^{L} \cdot\left(\tilde{r}_{i}\right)^{M} \cdot\left(\tilde{r}_{i}\right)^{R}\right) i=1 \ldots . n$ (see Figure 2) at the possible level $\alpha_{1}$, the following equations hold: [13]

$\pi\left(\tilde{r}_{1}+\cdots+\tilde{r}_{n} \leq b\right) \geq \alpha \quad$ if and only if

$(1-\alpha)\left(\left(\tilde{r}_{1}\right)^{L}+\cdots+\left(\tilde{r}_{n}\right)^{L}\right)+\alpha\left(\left(\tilde{r}_{1}\right)^{M}+\cdots+\left(\tilde{r}_{n}\right)^{M}\right) \leq b$

$\pi\left(\tilde{r}_{1}+\cdots+\tilde{r}_{n} \geq b\right) \geq \alpha \quad$ if and only if

$(1-\alpha)\left(\left(\tilde{r}_{1}\right)^{R}+\cdots+\left(\tilde{r}_{n}\right)^{R}\right)+\alpha\left(\left(\tilde{r}_{1}\right)^{M}+\cdots+\left(\tilde{r}_{n}\right)^{M}\right) \geq b$

Thus, with triangular fuzzy input and output numbers, the PCCR model converts to an LP model as follows (Lertworasirikul et al, 2003), (Peykani et al, 2019):

$$
\begin{aligned}
& (P C C R) \quad \max \quad \quad \quad \bar{f} \cdot \bar{f} \quad \bar{f} \\
& (1-\beta)\left(\tilde{y}_{q}^{t} v^{q}\right)^{R}-\beta\left(\tilde{y}_{q}^{t} v^{q}\right)^{M} \geq \bar{f} \\
& \left(1-\alpha_{\circ}\right)\left(\tilde{x}_{q}^{t} u^{q}\right)^{R}-\alpha \circ\left(\tilde{x}_{q}^{t} u^{q}\right)^{M} \geq 1 \\
& \left(1-\alpha_{\circ}\right)\left(\tilde{x}_{q}^{t} u^{q}\right)^{L}-\alpha \circ\left(\tilde{x}_{q}^{t} u^{q}\right)^{M} \leq 1 \\
& (1-\alpha)\left(\tilde{y}_{i}^{t} v^{q}-\tilde{x}_{i}^{t} u^{q}\right)^{L}-\alpha\left(\tilde{y}_{i}^{t} v^{q}-\tilde{x}_{i}^{t} u^{q}\right)^{M} \leq 1 \quad \text { for all } i=1 . \ldots . n \\
& u^{q} \geq \varepsilon_{r} \cdot v^{q} \geq \varepsilon_{s}
\end{aligned}
$$

\subsubsection{Integrated Necessity Theory and DEA Model}

In the possibility theory, there is another definable measure that uses the conjugate relation of possibility:

$$
\aleph(A \cap B)=\min (\aleph(A) \aleph(B))
$$

$\aleph$ is called the necessity measure, where $(\aleph(A)=1)$, indicating that $(\mathrm{A})$ is necessarily true. The dual relationship between the measures of possibility and necessity is the following:

$$
\pi(A)=1-\aleph(\bar{A}) . \forall A \subseteq U
$$

Also, the necessity measure provides the following conditions:

$$
\min (\aleph(A) \cdot \aleph(\bar{A}))=0
$$

The relationship between the possibility measure and the necessity measure satisfies the following:

$$
\begin{gathered}
\pi(A) \geq \aleph(A) \quad . \quad \forall A \subseteq U \\
\aleph(A)>0 \Rightarrow \pi(A)=1
\end{gathered}
$$


Efficiency Assessment Using Common-weight FDEA under a Multi-objective Approach as well as Possibility and Necessity Theory

$$
\pi(A)<1 \Rightarrow \aleph(A)=0
$$

The reference set $(\mathrm{U})$ is always considered finite.

Using the possibility distribution function, the necessity measure is calculated for set (A) of the reference set (U) as follows (Bandemer and Gottwald, 1995), (Klir and Yuan, 1996):

$$
\aleph(A)=\min _{i \in \bar{A}}\left\{1-\pi_{i}\right\}
$$

FDEA model is defined as the following model using fuzzy event necessity theory:

$$
\begin{array}{lll}
(N C C R) & \max & \text { u.v. } \bar{f}
\end{array}
$$

$\aleph\left(\tilde{y}_{q}^{t} v^{q} \geq \bar{f}\right) \geq \beta \quad(a)$

$\aleph\left(\left(\tilde{x}_{q}^{t} u^{q}=1\right) \geq \alpha\right.$ 。 $(b)$

$\aleph\left(\tilde{y}_{i}^{t} v^{q}-\tilde{x}_{i}^{t} u^{q} \leq 0\right) \geq \alpha \quad$ for all $i=1 \ldots . n(C)$

$u^{q} \geq \varepsilon_{r} \cdot v^{q} \geq \varepsilon_{s}$

In the above model, $\beta$ and $\alpha_{0} \in[0.1]$ are the assumed acceptable values for the constraints (a) and (b), respectively. Furthermore, $\alpha=\left[\alpha_{\circ} \ldots \ldots . \alpha_{n}\right]^{T} \in$ $[0 ; 1]^{n}$ is assumed a column of the allowable values vector for the possibility constraint (c).

If $\alpha$ is a member of $\beta . \alpha_{0} . \alpha_{1} \cdot \cdots . \alpha_{n}$, according to the dual relation between the possibility and necessity (13), the NCCR model can be solved similarly to the PCCR model (Lertworasirikul et al, 2003), (Peykani et al, 2019).

According to the fuzzy numbers ranking method, which was introduced for triangular fuzzy numbers $\tilde{r}_{i}=\left(\left(\tilde{r}_{i}\right)^{\mathrm{L}} \cdot\left(\tilde{r}_{i}\right)^{\mathrm{M}} \cdot\left(\tilde{r}_{i}\right)^{\mathrm{R}}\right)$ where $\mathrm{i}=1 \ldots . \mathrm{n}$ (Jimenez et al, 2007) and considering the possibility level $\alpha$ with $0 \leq \alpha \leq 1$, the following equations hold [21].

$$
\begin{aligned}
& \kappa\left(\tilde{r}_{1}+\cdots+\tilde{r}_{n} \leq \mathrm{b}\right) \geq \alpha \quad \text { if and only if } \\
& \alpha\left(\left(\tilde{r}_{1}\right)^{\mathrm{R}}+\cdots+\left(\tilde{r}_{n}\right)^{\mathrm{R}}\right)+(1-\alpha)\left(\left(\tilde{r}_{1}\right)^{\mathrm{M}}+\cdots+\left(\tilde{r}_{n}\right)^{\mathrm{M}}\right) \leq \mathrm{b} \\
& \kappa\left(\tilde{r}_{1}+\cdots+\tilde{r}_{n} \geq \mathrm{b}\right) \geq \alpha \quad \text { if and only if } \\
& \alpha\left(\left(\tilde{r}_{1}\right)^{\mathrm{L}}+\cdots+\left(\tilde{r}_{n}\right)^{\mathrm{L}}\right)+(1-\alpha)\left(\left(\tilde{r}_{1}\right)^{\mathrm{M}}+\cdots+\left(\tilde{r}_{n}\right)^{\mathrm{M}}\right) \geq \mathrm{b}
\end{aligned}
$$

\subsubsection{Triangular Fuzzy Numbers Ranking:}

An efficient way to compare fuzzy numbers is using a ranking function: $\Re F(R) \rightarrow R$, where $F(R)$ is considered as a set of fuzzy numbers and $R$ is the set of real numbers.

For every $\tilde{r}=\left(r_{1} \cdot r_{2} \cdot r_{3}\right) \in F(R)$, the function $\Re$ converts each fuzzy number to a real number by:

$\mathfrak{R}(\tilde{\mathrm{r}})=\left(\frac{\mathrm{r}_{1}+4 \mathrm{r}_{2}+\mathrm{r}_{3}}{6}\right)$

For both fuzzy numbers $\tilde{r}=\left(r_{1} \cdot r_{2} \cdot r_{3}\right)$ and $\tilde{t}=\left(t_{1} \cdot t_{2} \cdot t_{3}\right)$, a comparison is made as follows:

$$
\tilde{\mathrm{r}}>\tilde{\mathrm{t}} \quad \text { iff } \quad \mathfrak{R}(\tilde{\mathrm{r}})>\Re(\tilde{\mathrm{t}})
$$


Moosa Darijani, Maghsoud Amiri, Mohammad Taghi Taghavifard, Payam Hanafizadeh

$$
\begin{array}{lll}
\tilde{\mathrm{r}}<\tilde{\mathrm{t}} & \text { iff } & \Re(\tilde{\mathrm{r}})<\mathfrak{R}(\tilde{\mathrm{t}}) \\
\tilde{\mathrm{r}}=\tilde{\mathrm{t}} & \text { iff } & \mathfrak{R}(\tilde{\mathrm{r}})=\mathfrak{R}(\tilde{\mathrm{t}})
\end{array}
$$

A triangular fuzzy number $\tilde{\mathrm{r}}=\left(\mathrm{r}_{1} \cdot \mathrm{r}_{2} \cdot \mathrm{r}_{3}\right)$ is considered positive if $\Re(\tilde{\mathrm{r}})>0$ Where $\tilde{\mathrm{r}}>0$ if $\mathfrak{R}(\tilde{\mathrm{r}})>0$. Also, $\tilde{\mathrm{r}} \approx 0$ if $\Re(\tilde{\mathrm{r}})=0$ and $\tilde{\mathrm{r}} \prec 0$ if $\Re(\tilde{\mathrm{r}}) \prec 0$. If $\tilde{\mathrm{r}} \approx \tilde{\mathrm{t}}$, then the triangular fuzzy numbers $\tilde{\mathrm{r}}$ and $\tilde{\mathrm{t}}$ are considered equal (Sathish and Ganesan, 2011).

\subsection{Proposed Approach}

Now, suppose that there are $n$ DMUs with numbers of their input and output being equal. If each DMU has $r$ input and s output, then it can be defined as a vector of weights $u \in I R^{r}$ and $v \in I R^{s}$. Since input and output data are fuzzy numbers, $\tilde{x}_{i}^{t} u$ and $\tilde{y}_{i}^{t} v$ are used to show the input and output measures corresponding to the respective DMU.

Based on Equation (6) for fuzzy input and output with $p=1$ and $r+s+2$ and considering $3 \mathrm{n}$ constraints, the model can be written as the following LP model:

$$
\begin{aligned}
& \min \sum_{i=1}^{n}\left(\tilde{x}_{i}^{t} u-m\right)+\left(M-\tilde{y}_{i}^{t} v\right) \\
& m-\tilde{x}_{i}^{t} u \leq 0 \quad \text { for all } i=1 . \ldots . n \\
& M-\tilde{y}_{i}^{t} v \geq 0 \quad \text { for all } i=1 . \ldots . n \\
& \tilde{y}_{i}^{t} v-\tilde{x}_{i}^{t} u \leq 0 \quad \text { for all } i=1 . \ldots . n \\
& \sum_{i=1}^{r} u+\sum_{i=1}^{s} v=1 \\
& u \geq \varepsilon_{r} . v \geq \varepsilon_{s} \\
& M . m \geq 0
\end{aligned}
$$

In the above model, the values of $\varepsilon_{r}$ and $\varepsilon_{s}$ are the same as those in the CCR model (1). According to Equation (8), the model can be presented as follows: $\min \bar{f}$

$p\left(\sum_{i=1}^{n}\left(\tilde{x}_{i}^{t} u-m\right)+\left(M-\tilde{y}_{i}^{t} v\right) \leq \bar{f}\right) \geq \beta$

$p\left(m-\tilde{x}_{i}^{t} u \leq 0\right) \geq \alpha_{0} \quad$ for all $i=1 . \ldots . n$

$p\left(M-\tilde{y}_{i}^{t} v \geq 0\right) \geq \alpha_{1} \quad$ for all $i=1 \ldots . . n$

$p\left(\tilde{y}_{i}^{t} v-\tilde{x}_{i}^{t} u \leq 0\right) \geq \alpha_{2}$ for all $i=1 \ldots . n$

$\sum_{k=1}^{r} u+\sum_{k=1}^{s} v=1$

$u \geq \varepsilon_{r} \cdot v \geq \varepsilon_{s}$

M. $m \geq 0$

$\min \bar{f}$

The model can be simplified as follows:

$$
\begin{aligned}
& p\left(\sum_{i=1}^{n}\left(\tilde{x}_{i}^{t} u-\tilde{y}_{i}^{t} v\right) \leq \bar{f}+n m-n M\right) \geq \beta \\
& p\left(\tilde{x}_{i}^{t} u \geq m\right) \geq \alpha_{0} \quad \text { for all } i=1 . \ldots . n \\
& p\left(\tilde{y}_{i}^{t} v \leq M\right) \geq \alpha_{1} \quad \text { for all } i=1 . \ldots . n
\end{aligned}
$$


Efficiency Assessment Using Common-weight FDEA under a Multi-objective Approach as well as Possibility and Necessity Theory

$$
\begin{aligned}
& p\left(\tilde{y}_{i}^{t} v-\tilde{x}_{i}^{t} u \leq 0\right) \geq \alpha_{2} \text { for all } i=1 . \ldots . n \\
& \sum_{k=1}^{r} u+\sum_{k=1}^{s} v=1 \\
& M \geq \varepsilon_{r} \cdot v \geq \varepsilon_{s} \\
& M . m \geq 0
\end{aligned}
$$

If input and output numbers in the above model are convex and normal, according to Equation (9), the model should be written as:

$$
\min \bar{f}
$$

$$
\begin{aligned}
& \begin{array}{l}
\left.\sum_{i=1}^{n}\left(\tilde{x}_{i}^{t} u-\tilde{y}_{i}^{t} v\right)\right)_{\beta}^{L} \leq \bar{f}+n m-n M \\
\left(\tilde{x}_{i}^{t} u\right)_{\alpha_{\circ}}^{R} \geq m \quad \text { for all } i=1 . \ldots . n \\
\quad\left(\tilde{y}_{i}^{t} v\right)_{\alpha_{1}}^{L} \leq M \quad \text { for all } i=1 . \ldots . n \\
\quad\left(\tilde{y}_{i}^{t} v-\tilde{x}_{i}^{t} u\right)_{\alpha_{2}}^{L} \leq 0 \quad \text { for all } i=1 . \ldots . n
\end{array} \\
& \sum_{k=1}^{r} u+\sum_{k=1}^{v} v=1 \\
& \begin{array}{l}
u \geq \varepsilon_{r} . v \geq \varepsilon_{s} \\
M . m \geq 0
\end{array}
\end{aligned}
$$

Hence, when the input and output are triangular fuzzy numbers, the above PCCR model is defined through Equation (11) and can be shown by: $\min \bar{f}$

$$
\begin{aligned}
& (1-\beta)\left(\sum_{i=1}^{n}\left(\tilde{x}_{i}^{t^{L}} u-\tilde{y}_{i}^{t^{L}} v\right)\right)+\beta\left(\sum_{i=1}^{n}\left(\tilde{x}_{i}^{t^{M}} u-\tilde{y}_{i}^{t^{M}} v\right)\right) \leq \bar{f}+n m-n M \\
& \left(1-\alpha_{\circ}\right)\left(\tilde{x}_{i}^{t^{R}} u\right)+\alpha \circ\left(\tilde{x}_{i}^{t^{M}} u\right) \geq m \quad \text { for all } i=1 \ldots . . . n \\
& \left(1-\alpha_{1}\right)\left(\tilde{y}_{i}^{t^{L}} v\right)+\alpha_{1}\left(\tilde{y}_{i}^{t^{M}} v\right) \leq M \quad \text { for all } i=1 . \ldots . n \\
& \left(1-\alpha_{2}\right)\left(\tilde{y}_{i}^{t^{L}} v-\tilde{x}_{i}^{t^{L}} u\right)+\alpha_{2}\left(\tilde{y}_{i}^{t^{M}} v-\tilde{x}_{i}^{t^{M}} u\right) \leq 0 \quad \text { for all } i=1 . \ldots . n \\
& \sum_{k=1}^{r} u+\sum_{k=1}^{s} v=1 \\
& u \geq \varepsilon_{r} . v \geq \varepsilon_{s} \\
& M . m \geq 0
\end{aligned}
$$

Also, the input and output are substituted into Equation (14) to solve fuzzy models based on the necessity theory. Equation (17) is defined as follows:

$$
\begin{aligned}
& \min \bar{f} \\
& \aleph\left(\sum_{i=1}^{n}\left(\tilde{x}_{i}^{t} u-m\right)+\left(M-\tilde{y}_{i}^{t} v\right) \leq \bar{f}\right) \geq \beta \\
& \aleph\left(m-\tilde{x}_{i}^{t} u \leq 0\right) \geq \alpha_{0} \quad \text { for all } i=1 . \ldots . n \\
& \aleph\left(M-\tilde{y}_{i}^{t} v \geq 0\right) \geq \alpha_{1} \quad \text { for all } i=1 . \ldots . n \\
& \aleph\left(\tilde{y}_{i}^{t} v-\tilde{x}_{i}^{t} u \leq 0\right) \geq \alpha_{2} \quad \text { for all } i=1 . \ldots . n \\
& \sum_{k=1}^{r} u+\sum_{k=1}^{s} v=1 \\
& u \geq \varepsilon_{r} . v \geq \varepsilon_{s}
\end{aligned}
$$


Moosa Darijani, Maghsoud Amiri, Mohammad Taghi Taghavifard, Payam Hanafizadeh

M. $m \geq 0$

Thus, when triangular fuzzy numbers are used for input and output using Equation (15), we have:

$$
\begin{aligned}
& \min \bar{f} \\
& (1-\beta)\left(\sum_{i=1}^{n}\left(\tilde{x}_{i}^{t^{M}} u-\tilde{y}_{i}^{t^{M}} v\right)\right)+\beta\left(\sum_{i=1}^{n}\left(\tilde{x}_{i}^{t^{R}} u-\tilde{y}_{i}^{t^{R}} v\right)\right) \leq \bar{f}+n m-n M \\
& \left(1-\alpha_{\circ}\right)\left(\tilde{x}_{i}^{t^{M}} u\right)+\alpha_{\circ}\left(\tilde{x}_{i}^{t^{L}} u\right) \geq m \quad \text { for all } i=1 . \ldots . n \\
& \left(1-\alpha_{1}\right)\left(\tilde{y}_{i}^{t^{M}} v\right)+\alpha_{1}\left(\tilde{y}_{i}^{t^{R}} v\right) \leq M \quad \text { for all } i=1 . \ldots . n \\
& \left(1-\alpha_{2}\right)\left(\tilde{y}_{i}^{t^{M}} v-\tilde{x}_{i}^{t^{M}} u\right)+\alpha_{2}\left(\tilde{y}_{i}^{t^{R}} v-\tilde{x}_{i}^{t^{R}} u\right) \leq 0 \quad \text { for all } i=1 . \ldots . n \\
& \sum_{k=1}^{r} u+\sum_{k=1}^{s} v=1 \\
& u \geq \varepsilon_{r} \cdot v \geq \varepsilon_{s} \\
& M . m \geq 0
\end{aligned}
$$

In general, the proposed approach ranks the DMUs with input and output as triangular fuzzy numbers in three steps as follows:

Step 1: Model (17) is solved to find the weights of the input (minimization) and output (maximization) through Equation (21) for the possibility theory or Equation (23) for the necessity theory. $u^{*} \cdot v^{*}$

$E_{q}=\frac{\tilde{y}_{q}^{t} v^{*}}{\tilde{x}_{q}^{t} u^{*}}$

Step 2: Relation (24) is calculated to obtain the efficiency of each DMU.

Step 3: Relation (16) is used to rank efficiency values of $E_{q}$ for each DMU as fuzzy numbers.

\section{Numerical example:}

In this section, we use a practical example to test the proposed approach. The data used were adopted from the study (Mirhedayatian et al, 2012). The data were used to choose the best welding approaches to fixing nodular cast iron engine blocks. The study presented 11 different welding processes. Each of them had 10 separate criteria with two being the input criteria and eight the output criteria. The input and output were triangular fuzzy numbers. Welding processes were ranked using the proposed fuzzy DEA-TOPSIS hybrid method. The ranking welding processes are reported in Table 2.

Table 2. Ranking of welding processes using the DEA-TOPSIS approach

\begin{tabular}{|c|c|c|}
\hline $\begin{array}{c}\text { DM } \\
U\end{array}$ & Welding process & Rank \\
\hline 1 & SMAW & 3 \\
\hline 2 & FCAW & 7 \\
\hline
\end{tabular}


Efficiency Assessment Using Common-weight FDEA under a Multi-objective Approach as well as Possibility and Necessity Theory

\begin{tabular}{|c|c|c|}
\hline 3 & GMAW & 6 \\
\hline 4 & SAW & 9 \\
\hline 5 & GTAW & 4 \\
\hline 6 & PAW & 4 \\
\hline 7 & OFW & 2 \\
\hline 8 & EBW & 11 \\
\hline 9 & LBW & 10 \\
\hline 10 & Flame spray welding & 8 \\
\hline 11 & Furnace welding & 1 \\
\hline
\end{tabular}
level, i.e.,

In the present example, all fuzzy constraints have a specified possible

$\beta=\alpha_{0}=\cdots=\alpha_{n}$. Common weights are obtained for five different possible levels $(0,0.25,0.5,0.75$, and 1$)$. Then, the results of Welding Process Performance $\left(E_{q}\right)$ are evaluated by Relation (24) as presented in Table 3. The numbers are fuzzy

Table (3) Results of Welding Process Performance Evaluation Using Possibility-Common Weight DEA Model

Since the results calculated for each welding process are fuzzy numbers, triangular fuzzy numbers ranking approach (16) is used to compare these numbers, and the final ranking of the welding process is presented in Table 4 .

The NCCR approach used to rank the DMUs is pessimistic. The performance of each welding process is calculated using the necessity approach, with the results of the performance given in Table 5. Also, the ranking of the welding processes has been obtained using Relation (16), as reported in Table 6. The results obtained at different necessity and possibility levels are presented in Figure 3. These results show that when $\bar{f}$ is calculated for necessity, the trend of changes at different $\alpha$-levels in the necessity approach is the same as that in the possibility approach. Since the objective function is of minimum type, it is clear when the necessity level increases, which is also true for $\bar{f}$ value. The necessity approach is a pessimistic method; thus, the results are worse than the possibility approach findings.

\section{Conclusion}

The proposed model is a hybrid model composed of the common weights approach as well as the possibility theory and necessity theory and it was utilized to improve the FDEA for evaluating the performance of DMUs through fuzzy numbers as input and output according to the obtained results. The most obvious feature of the model is utilized when selecting the best DMU from different DMUs. In other words, this model can be used to select the best DMU. Also, if the results 
Moosa Darijani, Maghsoud Amiri, Mohammad Taghi Taghavifard, Payam Hanafizadeh

of this model are compared with previous models, it can be concluded that the present model enjoys a higher capability in evaluation of all DMUs. Since the common weight method was used in this model, the computational load was reduced dramatically. This model can be solved using other fuzzy number credibility approaches, the $\alpha$-level-based approach, tolerance approach, fuzzy arithmetic, fuzzy ranking approach, the fuzzy random/type-2 fuzzy, etc.

Table 3. Results of Welding Process Performance Evaluation Using Possibility-Common Weight DEA Model

\begin{tabular}{|c|c|c|c|c|c|c|}
\hline \multirow[t]{2}{*}{$\mathrm{DMU}$} & \multirow[t]{2}{*}{ Welding process } & \multicolumn{5}{|c|}{$E_{q}$ Possibility $\alpha^{\prime}-$ Level } \\
\hline & & $\alpha=0$ & $\alpha=0.25$ & $\alpha=0.5$ & $\alpha=0.75$ & $\alpha=1$ \\
\hline 1 & SMAW & $(0.333,0.573,1.293)$ & $(0.402,0.691,1.559)$ & $(0.452,0.820,1.924)$ & $(0.477,0.926,2.274)$ & $(0.514,1.000,2.456)$ \\
\hline 2 & FCAW & $(0.200,0.287,0.432)$ & $(0.242,0.346,0.520)$ & $(0.285,0.427,0.663)$ & $(0.368,0.565,0.894)$ & $(0.398,0.611,0.966)$ \\
\hline 3 & GMAW & $(0.201,0.288,0.433)$ & $(0.243,0.347,0.522)$ & $(0.286,0.428,0.665)$ & $(0.424,0.635,0.987)$ & $(0.458,0.687,1.067)$ \\
\hline 4 & SAW & $(0.142,0.190,0.258)$ & $(0.172,0.230,0.311)$ & $(0.211,0.294,0.409)$ & $(0.359,0.489,0.671)$ & $(0.388,0.529,0.726)$ \\
\hline 5 & GTAW & $(0.202,0.289,0.435)$ & $(0.243,0.348,0.523)$ & $(0.299,0.445,0.688)$ & $(0.506,0.738,1.125)$ & $(0.548,0.799,1.217)$ \\
\hline 6 & PAW & $(0.202,0.289,0.435)$ & $(0.243,0.348,0.523)$ & $(0.299,0.445,0.688)$ & $(0.506,0.738,1.125)$ & $(0.548,0.799,1.217)$ \\
\hline 7 & OFW & $(0.329,0.567,1.279)$ & $(0.398,0.685,1.544)$ & $(0.452,0.820,1.922)$ & $(0.476,0.924,2.267)$ & $(0.515,1.000,2.452)$ \\
\hline 8 & EBW & $(0.111,0.143,0.184)$ & $(0.134,0.172,0.222)$ & $(0.104,0.152,0.214)$ & $(0.279,0.367,0.480)$ & $(0.302,0.397,0.519)$ \\
\hline 9 & LBW & $(0.111,0.144,0.185)$ & $(0.134,0.73,0.223)$ & $(0.104,0.153,0.215)$ & $(0.280,0.368,0.480)$ & $(0.303,0.398,0.520)$ \\
\hline 10 & Flame spray weldin & $(0.144,0.93,0.261)$ & $(0.174,0.232,0.314)$ & $(0.214,0.297,0.413)$ & $(0.361,0.492,0.675)$ & $(0.391 .0 .532,0.730)$ \\
\hline 11 & Furnace welding & $(0.141,0.189,0.257)$ & $(0.171,0.229,0.310)$ & $(0.104,0.196,0.260)$ & $(0.301,0.422,0.590)$ & $(0.326,0.456,0.639)$ \\
\hline
\end{tabular}

Table 4. The final ranking of the welding Process Using the Possibility-Common Weight DEA Model

\begin{tabular}{|c|l|c|c|c|c|c|}
\hline \multirow{2}{*}{ DMU } & \multirow{2}{*}{ Welding process } & \multicolumn{5}{|c|}{$\alpha^{\prime}$-Level Ranking Possibility } \\
\cline { 3 - 7 } & & $\alpha=0$ & $\alpha=0.25$ & $\alpha=0.5$ & $\alpha=0.75$ & $\alpha=1$ \\
\hline 1 & SMAW & 1 & 1 & 1 & 1 & 1 \\
\hline 2 & FCAW & 5 & 5 & 5 & 5 & 5 \\
\hline 3 & GMAW & 4 & 4 & 4 & 4 & 4 \\
\hline 4 & SAW & 7 & 7 & 7 & 7 & 7 \\
\hline 5 & GTAW & 3 & 3 & 3 & 3 & 3 \\
\hline 6 & PAW & 3 & 3 & 3 & 3 & 3 \\
\hline 7 & OFW & 2 & 2 & 2 & 2 & 2 \\
\hline
\end{tabular}


Efficiency Assessment Using Common-weight FDEA under a Multi-objective Approach as well as Possibility and Necessity Theory

\begin{tabular}{|c|l|c|c|c|c|c|}
\hline 8 & EBW & 10 & 10 & 10 & 10 & 10 \\
\hline 9 & LBW & 9 & 9 & 9 & 9 & 9 \\
\hline 10 & Flame spray welding & 6 & 6 & 6 & 6 & 6 \\
\hline 11 & Furnace welding & 8 & 8 & 8 & 8 & 8 \\
\hline
\end{tabular}

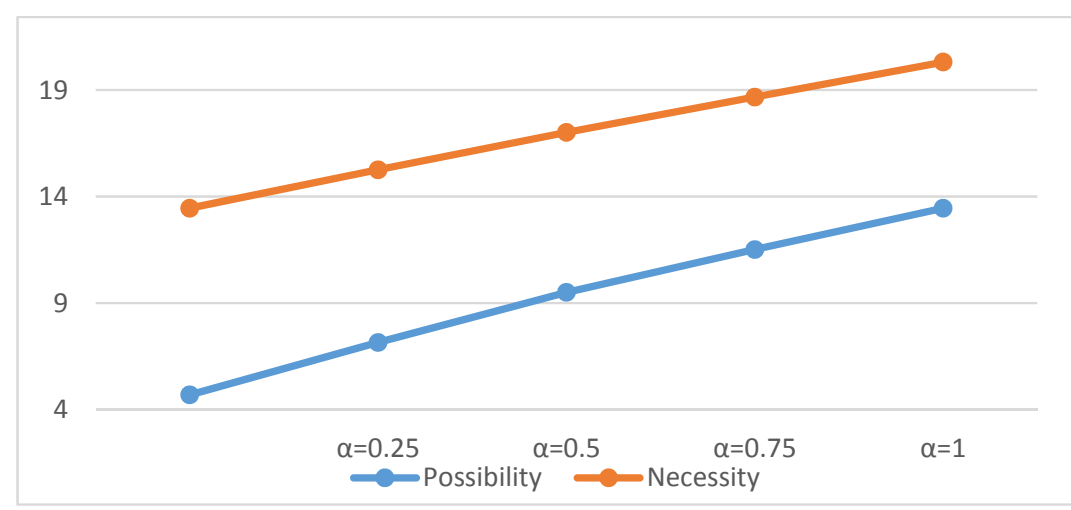

Figure 3. Comparative graph of the trend of changes $\bar{f}$ at different levels of Possibility and Necessity

Table 5. Results of Welding Process Performance Evaluation Using Necessity Common Weight DEA Model

\begin{tabular}{|l|l|c|c|c|c|c|}
\hline \multirow{2}{*}{ DMU } & \multirow{2}{*}{ Welding process } & \multicolumn{5}{|c|}{$E_{q}$ Necessity $\alpha$-Level } \\
\cline { 3 - 7 } & & $\alpha=0$ & $\alpha=0.25$ & $\alpha=0.5$ & $\alpha=0.75$ & $\alpha=1$ \\
\hline 1 & SMAW & $(0.514,0.999,2.454)$ & $(0.514,1.053,2.670)$ & $(0.540,1.103,2.791)$ & $(0.566,1.149,2.899)$ & $(0.589,1.191,2.997)$ \\
\hline 2 & FCAW & $(0.397,0.610,0.966)$ & $(0.400,0.608,0.932)$ & $(0.359,0.534,0.788)$ & $(0.369,0.548,0.807)$ & $(0.379,0.561,0.824)$ \\
\hline 3 & GMAW & $(0.458,0.686,1.066)$ & $(0.503,0.734,1.092)$ & $(0.459,0652,0.933)$ & $(0.4690 .666,0.951)$ & $(0.478,0.678,0.967)$ \\
\hline 4 & SAW & $(0.388,0.529,0.725)$ & $(0.461,0.616,0.826)$ & $(0.428,0.565,0.745)$ & $(0.436,0.575,0.758)$ & $(0.443,0.584,0.770)$ \\
\hline 5 & GTAW & $(0.5470 .798,1.216)$ & $(0.708,1.026,1.556)$ & $(0.724,1.051,1.597)$ & $(0.7391 .074,1.633)$ & $(0.753,1.095,1.665)$ \\
\hline 6 & PAW & $(0.5470 .798,1.216)$ & $(0.708,1.026,1.556)$ & $(0.724,1.051,1.597)$ & $(0.7391 .074,1.633)$ & $(0.753,1.095,1.665)$ \\
\hline 7 & OFW & $(0.5150 .999,0.2451)$ & $(0.597,1.040,2.027)$ & $(0.687,1.060,1.745)$ & $(0.706,1.087,1.780)$ & $(0.724,1.110,1.811)$ \\
\hline 8 & EBW & $(0.302,0.397,0.519)$ & $(0.391,0.511,0.664)$ & $(0.328,0.422,0.589)$ & $(0.330,0.446,0.596)$ & $(0.331,0.449,0.601)$ \\
\hline 9 & LBW & $(0.303,0.398,0.519)$ & $(0.392,0.511,0.665)$ & $(0.328,0.433,0.589)$ & $(0.330,0.447,0.596)$ & $(0.331,0.450,0.602)$ \\
\hline 10 & Flame spray welding & $(0.391,0.532,0.729)$ & $(0.505,0.684,0.933)$ & $(0.517,0.701,0.958)$ & $(0.528,0.716,0.979)$ & $(0.538,0.730,0.999)$ \\
\hline 11 & Furnace welding & $(0.326,0.456,0.639)$ & $(0.544,0.777,1.135)$ & $(0.671,1.064,1.816)$ & $(0.685,1.095,1.890)$ & $(0.698,1.124,1.961)$ \\
\hline
\end{tabular}


Moosa Darijani, Maghsoud Amiri, Mohammad Taghi Taghavifard, Payam Hanafizadeh

Table 6. The final ranking of the welding Process Using the necessity Common Weight DEA Model

\begin{tabular}{|l|l|c|c|c|c|c|}
\hline \multirow{2}{*}{ DMU } & \multirow{2}{*}{ Welding process } & \multicolumn{5}{|c|}{ Ranking Necessity $\alpha$-Level } \\
\cline { 3 - 7 } & & $\alpha=0$ & $\alpha=0.25$ & $\alpha=0.5$ & $\alpha=0.75$ & $\alpha=1$ \\
\hline 1 & SMAW & 1 & 1 & 1 & 1 & 1 \\
\hline 2 & FCAW & 5 & 7 & 8 & 8 & 8 \\
\hline 3 & GMAW & 4 & 5 & 6 & 6 & 6 \\
\hline 4 & SAW & 7 & 8 & 7 & 7 & 7 \\
\hline 5 & GTAW & 3 & 3 & 4 & 4 & 4 \\
\hline 6 & PAW & 3 & 3 & 4 & 4 & 4 \\
\hline 7 & OFW & 2 & 2 & 3 & 3 & 3 \\
\hline 8 & EBW & 10 & 10 & 10 & 10 & 10 \\
\hline 9 & LBW & 9 & 9 & 9 & 9 & 9 \\
\hline 10 & Flame spray welding & 6 & 6 & 5 & 5 & 5 \\
\hline 11 & Furnace welding & 8 & 4 & 2 & 2 & 2 \\
\hline
\end{tabular}

\section{REFERENCES}

[1] Bandemer,H. \& Gottwald, S. (1995), Fuzzy Sets, Fuzzy Logic, Fuzzy Methods. Chichester: Wiley;

[2] Carrillo, Marianela \& Jorge, Jesús M (2016), A Multi-objective DEA Approach to Ranking Alternatives. Expert Systems with Applications, 50, 130139;

[3] Charnes, A., Cooper, W. W. \& Rhodes, E. L. (1978), Measuring the Efficiency of Decision Making Units. European Journal of Operational Research, 2, 444-429;

[4] Dubois, D. \& Prade, H. (1988), Possibility Theory: Qualitative and Quantitative Aspects. Handbook of Defeasible Reasoning and Uncertainty Management Systems, 169-226;

[5] Dubois, D. \& Prade, H. (2001), Possibility Theory, Probability Theory and Multiple-Valued Logics: A Clarification. Annals of Mathematics and Artificial Intelligence, 32(1-4), 35-66;

[6] Dyson, R. G. \& Thanassoulis, E. (1988), Reducing Weight Flexibility in Data Envelopment Analysis. Journal of the Operational Research Society, 39(6), 563576 ; 
Efficiency Assessment Using Common-weight FDEA under a Multi-objective Approach as well as Possibility and Necessity Theory

[7] Emrouznejad, A., Tavana, M. \& Hatami-Marbini, A. (2014), The State of the Art in Fuzzy Data Envelopment Analysis. Studies in Fuzziness and Soft Computing Envelopment Analysis, 1-46;

[8] Hosseinzadeh Lofti, F., Jahanshahloo, G. R., Khodabakhshi, M., RostamyMalkhlifeh, M., Moghaddas, Z. \& Vaez-Ghasemi, M. (2013), A Review of Ranking Models in Data Envelopment Analysis. Journal of Applied Mathematics, 20ArticleID492421;

[9] Jahanshahloo, G. R., Lotfi, F. H., Khanmohammadi, M., Kazemimanesh, M. \& Rezaie, V. (2010), Ranking of Units by Positive Ideal DMU with Common Weights. Expert Systems with Applications, 37, 7483-7488;

[10] Jimenez, M., Arenas, M., Bilbao, A. \& Rodriguez, M. V. (2007), Linear Programming with Fuzzy Parameters: An Interactive Method Resolution. European Journal of Operational Research 177, 1599-1609;

[11] Kao, C. \& Hung, H. (2005), Data Envelopment Analysis with Common Weights. The Compromise Solution Approach. Journal of the Operational Research Society, 56, 1196-1203;

[12] Klir, G. J. \& Yuan, B. (1996), Fuzzy Sets and Fuzzy Logic: Theory and Applications. Possibility Theory versus Probab. Theory, 32(2), 207-208;

[13] Kheirollahi, Hooshang, Hessari, Peyman, Charles, Vincent \& Chawshini, Rasoul (2017), An Input Relaxation Model for Evaluating Congestion in Fuzzy DEA. Croatian Operational Research Review, 8(2), 391-408;

[14] Lertworasirikul, S., Fang, S.-C., Joines, J. A. \& Nuttle, H. L. W. (2003), Fuzzy Data Envelopment Analysis (DEA): A Possibility Approach. Fuzzy Sets and Systems, 139, 379-394;

[15] Li, X.-Y. \& Cui, J.-C. (2007), Extra Resource Allocation Problem with the Most Compromise Common Weights Based On DEA. The First International Symposium on Optimization and Systems Biology;

[16] Liu, B. (1999), Uncertain Programming. A Wiley-Interscience Publication, New York;

[17] Mirhedayatian, S. M., Vahdat, S. E., Jafarian Jelodar, M. \& Farzipoor Saen, R. (2012), Welding Process Selection for Repairing Nodular Cast Iron Engine Block by Integrated Fuzzy Data Envelopment Analysis and TOPSIS Approaches. Materials and Design, 43(2013), 22-282;

[18] Peykani, Pejman, Mohammadi, Emran, Emrouznejad, Ali, Pishvaee, Mir Saman, \& Rostamy-Malkhalifeh, Mohsen (2019), Fuzzy Data Envelopment Analysis: An Adjustable Approach. Expert Systems with Applications, 136, 439452;

[19] Roll, Y., Cook, W. D. \& Golany, B. (1991), Controlling Factor Weights in Data Envelopment Analysis. IIE Transactions 23(1); 
Moosa Darijani, Maghsoud Amiri, Mohammad Taghi Taghavifard, Payam Hanafizadeh

[20] Sathish, S. \& k.Ganesan. (2011), A Simple Approach to Fuzzy Critical Path Analysis in Project Networks. International Journal Scientific \&Engineering Research, 2(12);

[21] Zahhiri, B., Tavakkoli-Moghadam, R. \& Pishvaee, M. S. (2014), A Robust Possibilistic Programming Approach to Multi-Period Location-Allocation of

Organ Transplant Centers Under Uncertainty. Computer\& Industrial

Engineering, 74, 139-148;

[22] Zimmermann, H. J. (1996), Fuzzy Set Theory and Its Application. Kluwer Academic Publishers. 